\title{
ChemComm
}

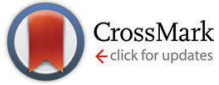

Cite this: Chem. Commun., 2016, 52,8854

Received 13th April 2016, Accepted 15th June 2016

DOI: $10.1039 / c 6 c c 03076 a$

www.rsc.org/chemcomm

\section{Photosensitizer anchored gold nanorods for targeted combinational photothermal and photodynamic therapy $\dagger$}

\author{
Huijun Phoebe Tham, ${ }^{\text {abc }}$ Hongzhong Chen, ${ }^{b}$ Yu Hui Tan, ${ }^{b}$ Qiuyu Qu, ${ }^{b}$ \\ Sivaramapanicker Sreejith, ${ }^{\mathrm{b}}$ Lingzhi Zhao, ${ }^{\mathrm{b}}$ Subbu S. Venkatraman* ${ }^{\star \mathrm{c}}$ and \\ Yanli Zhao*bc
}

\begin{abstract}
Silylated zinc phthalocyanine ( $\mathrm{ZnPc}$ ) was anchored onto silicacoated gold nanorods (AuNR) with retained local surface plasmon resonance (LSPR). Independent LSPR and singlet oxygen production of anchored $\mathrm{ZnPc}$ enhance the photothermal and photodynamic efficacy of the obtained AuNR-Si-ZnPc under NIR light excitation. AuNR-Si-ZnPc was further grafted with hyaluronic acid (HA). Since HA has selective targeting capability to CD44 antigens, the final hybrid could target cancer cells directly for synergistic photothermal and photodynamic therapy.
\end{abstract}

Photodynamic therapy (PDT) and photo-thermal therapy (PTT) are viable alternatives to traditional cancer therapeutic approaches. PDT is minimally invasive, yet highly localized. ${ }^{1}$ It exhibits minimal dark cytotoxicity with negligible systemic effects. ${ }^{2}$ Singlet oxygen $\left({ }^{1} \mathrm{O}_{2}\right)$ is the most prevalent reactive oxygen species produced upon the activation of photosensitizers to cause cell death. ${ }^{3}$ Photosensitizers can be delivered through mesoporous silica nanoparticles ${ }^{4}$ and ultrasmall polymer dots. ${ }^{5}$ In terms of PTT, photothermal agents such as porphyrins ${ }^{6}$ and polydopamine ${ }^{7}$ were often used. However, these agents are prone to be photobleaching with a relatively low absorption coefficient. ${ }^{8}$ Both PDT and PTT are initiated by light irradiation with a suitable wavelength. The combination of PDT and PTT has been proven to have a synergistic therapeutic effect. ${ }^{9-14}$ This combinational approach could be performed using the same activation light source, while PDT and PTT work independently of and complementarily to each other. ${ }^{15}$ Some studies have been carried out on gold nanorods (AuNR) for combined PDT and PTT. ${ }^{16-18}$ However, these studies indicate complicated photosensitizer

\footnotetext{
${ }^{a}$ Interdisciplinary Graduate School, Nanyang Technological University, 50 Nanyang Avenue, 639798, Singapore

${ }^{b}$ Division of Chemistry and Biological Chemistry, School of Physical and Mathematical Sciences, Nanyang Technological University, 21 Nanyang Link, 637371, Singapore.E-mail: zhaoyanli@ntu.edu.sg

${ }^{c}$ School of Materials Science and Engineering, Nanyang Technological University, 50 Nanyang Avenue, 639798, Singapore. E-mail: assubbu@ntu.edu.sg

$\dagger$ Electronic supplementary information (ESI) available: Additional synthesis and characterization data. See DOI: 10.1039/c6cc03076a
}

release mechanisms, ${ }^{18}$ use relatively weak photosensitizers, ${ }^{16,17}$ and are non-targeted. ${ }^{16}$ Only a few studies have been reported using a single light source to activate both PTT and PDT. ${ }^{17}$ Thus, developing a nanosystem capable of harnessing combinational PTT and PDT by responding to a single light source to address abovementioned drawbacks is still a challenge. In this work, photosensitizer anchored AuNR for combinational PTT and PDT was prepared to address this issue.

Gold in the nanoscale range exhibits localized surface plasmon resonance (LSPR), ${ }^{19}$ leading to a rapid temperature increase locally, increasing blood flow, and attracting more oxygen for PDT. ${ }^{20}$ An AuNR has two absorption bands, with the one corresponding to the longitudinal axis of the rod easily tunable. The AuNR is thus an attractive nanocarrier, a photothermal agent and a viable imaging agent. When the absorption wavelength of a photosensitizer and the AuNR overlaps, the LSPR can enhance the absorption coefficient of the photosensitizer, further improving PDT. ${ }^{21}$ In this case, Li et al. successfully utilized AuNR for photo-induced drug release along with photothermal therapy. ${ }^{22}$

To this end, tetraamino zinc phthalocyanine $\left(\mathrm{ZnPc}-4 \mathrm{NH}_{2}\right)$ was employed as a PDT precursor for its easy functionalization. 3-(Triethoxysilyl)propyl isocyanate was reacted with $\mathrm{ZnPc}-4 \mathrm{NH}_{2}$ to give silica modified ZnPc (ZnPc-Si, Fig. 1). After the tetrasubstitution, the $\mathrm{Q}$ band of $\mathrm{ZnPc}$ red-shifted to $716 \mathrm{~nm}$. The complete reaction of the isocyanate group of 3-(triethoxysilyl)propyl isocyanate was confirmed by the disappearance of the -OCN peak at $2270 \mathrm{~cm}^{-1}$ in the FTIR spectra (Fig. S1, ESI $\dagger$ ).

Cetyltrimethylammonium bromide (CTAB)-stabilized AuNR used in this project were synthesized using the seed-mediated method. ${ }^{23}$ It showed the absorption peak at $700 \mathrm{~nm}$, optically matching that of ZnPc. CTAB on the surface was then subjected to a ligand exchange with 11-mercaptoundecanoic acid (MUA) on account of its strong capability for the $\mathrm{Au}-\mathrm{S}$ bond formation. This ligand replacement ensures the minimum cytotoxicity, as toxic CTAB was removed. MUA stabilizes AuNR in ethanol, which is necessary for the formation of a silica layer on the surface via the well-known Stöber method. ${ }^{24}$ The silica layer 

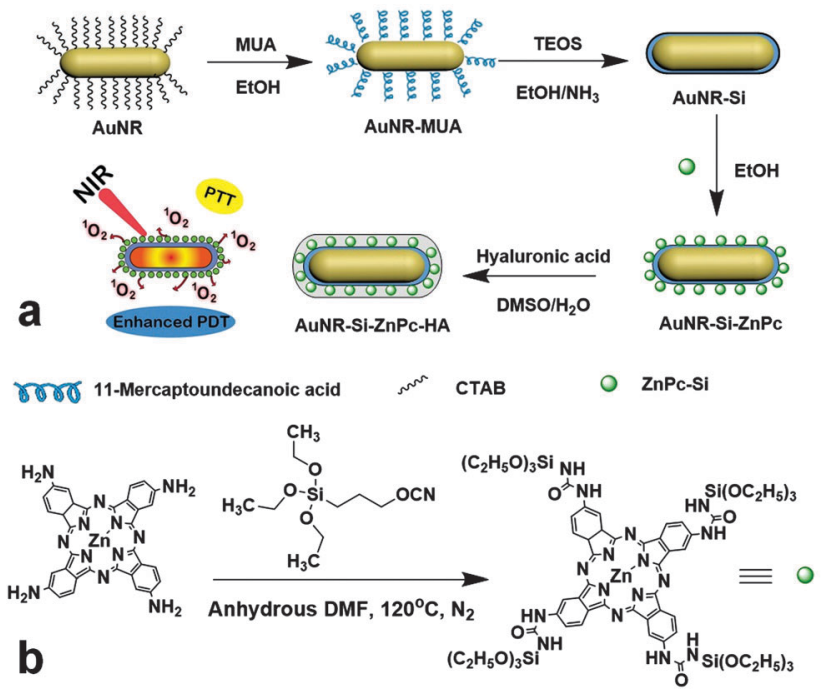

Fig. 1 Schematic illustration of (a) the formation of AuNR-Si-ZnPc-HA and (b) the synthesis of the ZnPc-Si precursor.

was used to minimize any possible electron transfer from ZnPc to AuNR, which could otherwise reduce the activity of ZnPc. ${ }^{18}$ ZnPc-Si in anhydrous dimethylformamide (DMF) solution was directly used to graft onto the silica coated AuNR (AuNR-Si) using a method similar to one that was previously reported. ${ }^{25}$ Since hyaluronic acid (HA) could target over-expressed CD44 receptors of many cancer cell lines often involved in metastasis, ${ }^{26}$ and also provide hydrophilicity to improve the dispersity of the functionalized AuNR in water, it was integrated through electrostatic interaction as the last step to yield the final hybrid 711-AuNR-Si-ZnPc-HA (see Fig. 1a).

The preparation process was monitored by transmission electron microscopy (TEM, Fig. 2). The length and width of the obtained bare AuNR were measured to be $51 \pm 2 \mathrm{~nm}$ and $15 \pm 3 \mathrm{~nm}$, respectively, with a calculated aspect ratio of about 3.4. The thickness of the initial silica coating was measured to be about $4 \pm 1 \mathrm{~nm}$. After grafting $\mathrm{ZnPc}-\mathrm{Si}$, the thickness of this layer increased to about $12 \pm 1 \mathrm{~nm}$. After finally coating with a layer of HA, the total coating thickness was $15 \pm 2 \mathrm{~nm}$. The successive coatings were also analyzed by observing zeta potential and absorbance changes (Fig. S2, ESI $\dagger$ ).

The successful incorporation of $\mathrm{ZnPc}$ on the surface of AuNR-Si was confirmed by UV-vis and fluorescence spectra (Fig. S3a and b, ESI $\dagger$ ). The characteristic Soret band of ZnPc at $350 \mathrm{~nm}$ in the spectrum of AuNR-Si-ZnPc indicates the presence of ZnPc. The peak did not match exactly with that of free ZnPc-Si due to the interaction between AuNR-Si and ZnPc. ${ }^{9}$ The Q band of ZnPc-Si in AuNR-Si-ZnPc was not observable due to the spectral overlap at around 700-720 nm. Upon excitation at $650 \mathrm{~nm}$, the emission peak of AuNR-Si-ZnPc at $730 \mathrm{~nm}$ corresponds to that of $\mathrm{ZnPc}$, although slight quenching was observed due to the interaction between AuNR and ZnPc. Thus, the silica layer was sufficient to prevent the total quenching of $\mathrm{ZnPc}$ in the hybrid. ${ }^{15}$ The generation of ${ }^{1} \mathrm{O}_{2}$ from 711-AuNR-SiZnPc-HA upon irradiation by NIR light was studied by using
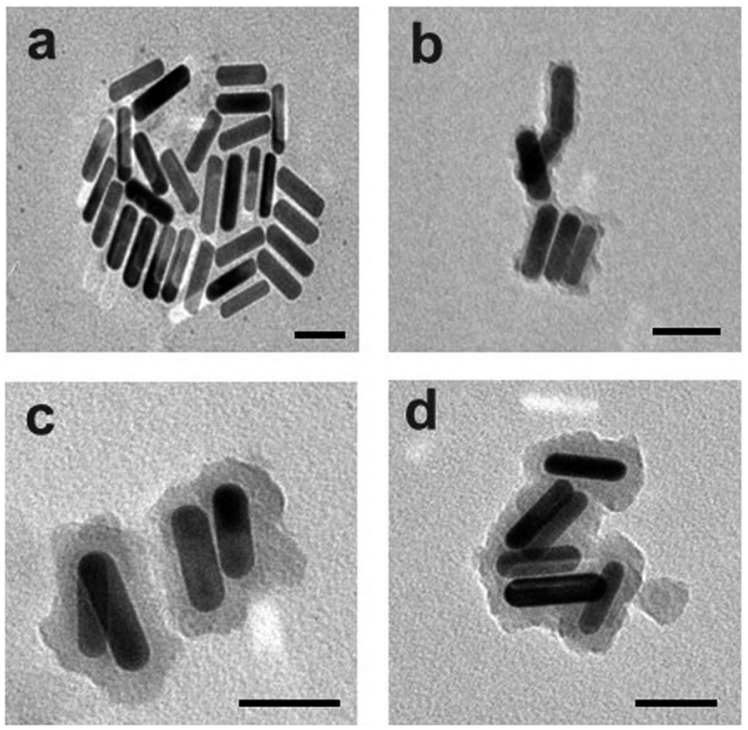

Fig. 2 TEM images of (a) $700 \mathrm{~nm}$ AuNR, (b) AuNR-Si, (c) AuNR-Si-ZnPc, and (d) 711-AuNR-Si-ZnPc-HA. Scale bar $=50 \mathrm{~nm}$.

9,10-anthracenediyl-bis-(methylene)dimalonic acid (ABDA). ABDA reacts readily with ${ }^{1} \mathrm{O}_{2}$ to produce an endoperoxide, and this process can be detected by measuring the absorbance changes. It can be seen that the ABDA absorption peaks were slowly quenched over time due to the ${ }^{1} \mathrm{O}_{2}$ generation by 711-AuNR-SiZnPc-HA (Fig. S3c, ESI $\dagger$ ).

In order to determine the photothermal capability, 711-AuNRSi-ZnPc-HA and bare AuNR solutions were irradiated using a $730 \mathrm{~nm}$ laser at a power intensity of $2.14 \mathrm{~W} \mathrm{~cm}^{-2}$. The temperature of the bare AuNR solution increased by about $23{ }^{\circ} \mathrm{C}$, and that of 711-AuNR-Si-ZnPc-HA increased by $21{ }^{\circ} \mathrm{C}$. The temperature of water did not change appreciably. The concentration-dependent temperature change of 711-AuNR-Si-ZnPc-HA was also measured (Fig. S4, ESI $\dagger$ ). The photostability of 711-AuNR-Si-ZnPc-HA was tested, showing good stability even after $30 \mathrm{~min}$ irradiation (Fig. S5a and b, ESI $\dagger$ ). The photothermal stability of 711-AuNRSi-ZnPc-HA was also tested, presenting over $97 \%$ stability after 4 cycles with 5 min irradiation at $2 \mathrm{~W} \mathrm{~cm}^{-2}$ each cycle (Fig. S5c, ESI $\dagger$ ). The hydrodynamic size of 711-AuNR-Si-ZnPc-HA was measured in water and phosphate buffer solution (PBS) for $48 \mathrm{~h}$, also showing good stability (Fig. S5d and e, ESI $\dagger$ ).

For the PDT determination, HA functionalized solid silica nanoparticles (Si-ZnPc-HA) were prepared through the same procedure as the PDT-only control (Fig. S6, ESI $\dagger$ ), and 711AuNR-Si-HA without ZnPc was used as the PTT-only control. The coatings of ZnPc-Si and HA on silica nanoparticles were analyzed using zeta potential (Fig. S7, ESI $\dagger$ ). The amount of $\mathrm{ZnPc}$ grafted was calculated to be about $5.7 \times 10^{15}$ molecules per mg of Si-ZnPc in order to maintain a content similar to that on AuNR-Si-ZnPc (ca. 15.4 $\mu \mathrm{g}$ ZnPc per mg of AuNR-Si-ZnPc). These values were calculated from the ZnPc-Si calibration curve $\left(r^{2}=0.9975\right)$. The peaks in the fluorescence spectra of Si-ZnPc at about $709 \mathrm{~nm}$ and $639 \mathrm{~nm}$ corresponding to the Q bands of ZnPc-Si molecules indicate that ZnPc-Si was successfully grafted onto the Si nanoparticles (Fig. S8, ESI $\dagger$ ). 711-AuNR-Si-HA was 
synthesized in an identical way to 711-AuNR-Si-ZnPc-HA without grafting of ZnPc-Si.

The targeting ability of HA was investigated using confocal laser scanning microscopy, flow cytometry and inductively coupled plasma optical emission spectrometry (ICP-OES). HeLa cells were used for their high expression of CD44 receptors. MCF-7 was used as the negative control as it has much lower expression of CD44 receptors. After $24 \mathrm{~h}$ of incubation with 711-AuNR-Si-ZnPc-HA, it was obvious that the internalization of the hybrid in HeLa cells was much higher than that in MCF-7 (Fig. $3 \mathrm{a}$ and b). In the flow cytometry studies, upon the introduction of 711-AuNR-Si-ZnPc-HA and AuNR-Si-ZnPc-NH $\mathrm{N}_{2}$ into HeLa cells, the cells showed higher uptake of the HA-coated nanosystem (711-AuNR-Si-ZnPc-HA) than that without HA (AuNR-Si-ZnPc- $\mathrm{NH}_{2}$ ), as demonstrated by the enhanced fluorescence intensity (Fig. 3c). This observation was very different to the MCF-7 cells where the fluorescence intensity of 711-AuNR-SiZnPc-HA and AuNR-Si-ZnPc-NH ${ }_{2}$ was similar after the incubation (Fig. 3d). Thus, the specificity of HA towards CD44 receptors was demonstrated. ICP-OES analyses further indicate that HeLa cells could internalize the amount of the hybrid 3 times higher than MCF-7 cells (Fig. S9, ESI $\dagger$ ).

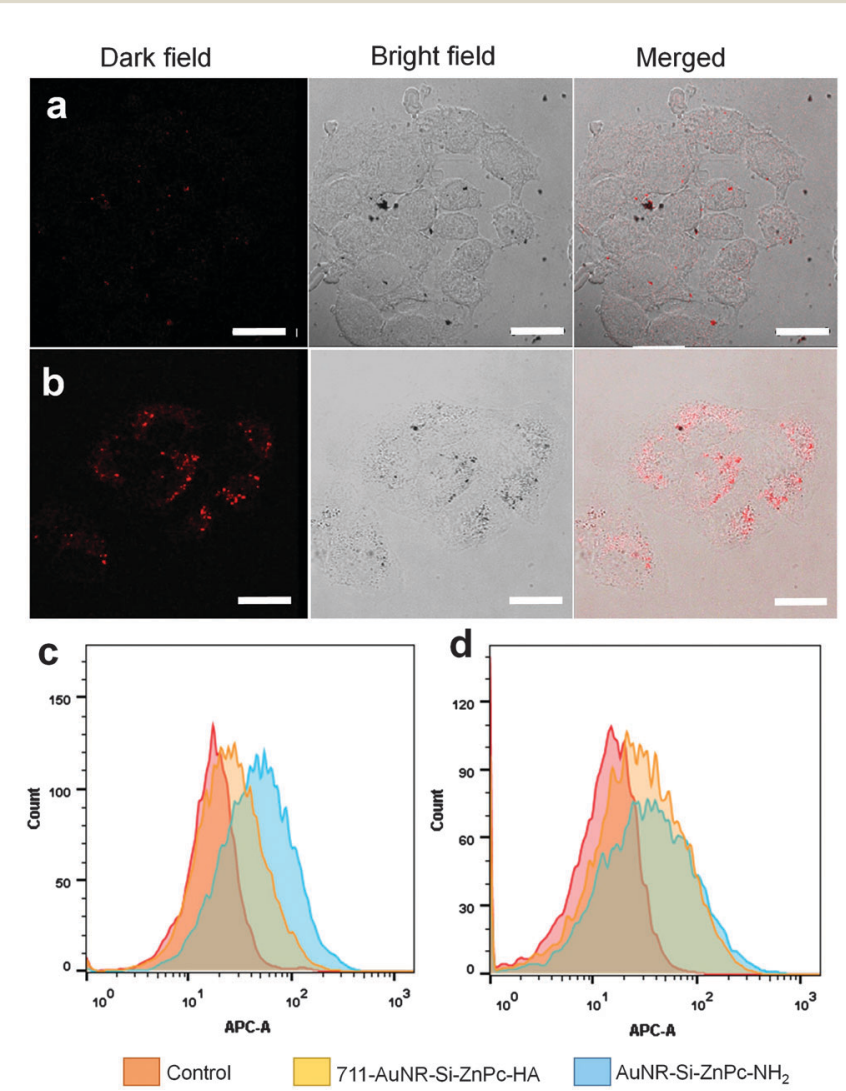

Fig. 3 Targeting ability of 711-AuNR-Si-ZnPc-HA on (a) MCF-7 and (b) HeLa cells after $24 \mathrm{~h}$ incubation. Cells were fed with 711-AuNR-Si-

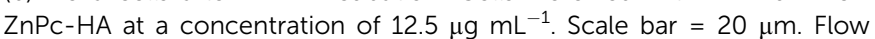
cytometry histograms of (c) HeLa cells and (d) MCF-7 with the addition of 711-AuNR-Si-ZnPc-HA containing the targeting group and AuNR-Si$\mathrm{ZnPc}-\mathrm{NH}_{2}$ as the non-targeting control. Cells were incubated at a concentration of $50 \mu \mathrm{g} \mathrm{mL}^{-1}$ for $4 \mathrm{~h}$.
The toxicity of the synthesized hybrid was investigated using 3-(4,5-dimethylthiazol-2-yl)-2,5-diphenyl tetrazolium bromide (MTT) assay. After incubating for 24 and 48 hours in the dark and testing using MTT, the absorbance of the solubilized formazan product was measured using a microplate reader at $570 \mathrm{~nm}$. It can be seen (Fig. S10, ESI $\dagger$ ) that 711-AuNR-SiZnPc-HA exhibited the minimal cytotoxicity at concentrations up to $180 \mu \mathrm{g} \mathrm{mL} \mathrm{mL}^{-1}$ after $48 \mathrm{~h}$ of incubation, indicating its excellent biocompatibility. The concentrations of ZnPc grafted onto silica nanoparticles and AuNR samples added were equivalent. The concentrations of gold in AuNR-Si-HA and 711-AuNR-SiZnPc-HA were also equivalent. Cells were incubated with these samples for 24 hours before photoirradiation. For MCF-7 cells, after irradiation, the cell viability showed a slight decrease to $83.7 \%$ when treated with Si-ZnPc-HA at the highest concentration. When fed with 711-AuNR-Si-ZnPc-HA under irradiation, the cell viability decreased to $52 \%$ (Fig. $4 \mathrm{c}$ ). For HeLa cells, the cellkilling effect was much more pronounced due to the targeting ability of HA. When incubated with 711-AuNR-Si-ZnPc-HA under laser irradiation, the cell viability decreased significantly to $21 \%$ (Fig. 4b). Non-targeted control experiments were also conducted using samples (Si-ZnPc, AuNR-Si-ZnPc, and AuNR-Si) without HA coating. For HeLa cells, there was a marked increase in the killing effect of the samples with $\mathrm{HA}$ as compared to those without HA coating (Fig. 4b). For MCF-7 cells, the killing effect of those with HA coating $v s$. those without HA was less pronounced (Fig. 4c), further confirming the targeting ability of 711-AuNR-Si-ZnPc-HA towards CD44 receptor overexpressed cancer cell lines.
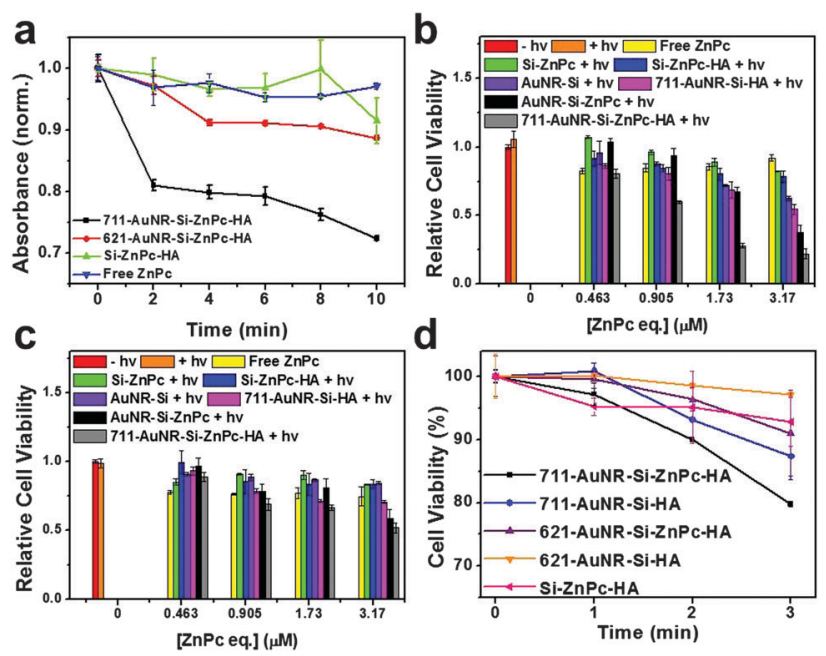

Fig. 4 (a) Relative absorbance of ABDA at $380 \mathrm{~nm}$ in the presence of free ZnPc, Si-ZnPc-HA, 621-AuNR-Si-ZnPc-HA and 711-AuNR-Si-ZnPc-HA upon laser irradiation (730 nm, $0.535 \mathrm{~W} \mathrm{~cm}^{-2}$ ) over time. (b and c) Phototoxicity of free $\mathrm{ZnPc}, \mathrm{Si}-\mathrm{ZnPc}$, AuNR-Si and AuNR-Si-ZnPc with and without HA coating on (b) HeLa cells and (c) MCF-7 cells. Cells were irradiated using a $730 \mathrm{~nm} 0.535 \mathrm{~W} \mathrm{~cm}^{-2}$ laser for $10 \mathrm{~min}$. (d) Phototoxicity of cells incubated with 621-AuNR-Si-ZnPc-HA, 621-AuNR-Si-HA, 711-AuNR$\mathrm{Si}-\mathrm{ZnPc}-\mathrm{HA}$, 711-AuNR-Si-HA, and Si-ZnPc-HA with similar gold and $\mathrm{ZnPc}$ concentrations under $730 \mathrm{~nm}, 0.535 \mathrm{~W} \mathrm{~cm}^{-2}$ laser irradiation for $0,1,2$ and $3 \mathrm{~min}$. A concentration of $20 \mu \mathrm{g} \mathrm{mL}^{-1}$ was used, and the incubation for $24 \mathrm{~h}$ was performed prior to the irradiation. 
To prove that the LSPR effect could improve the PDT efficacy, AuNR that absorb at $621 \mathrm{~nm}$ were further synthesized, and $\mathrm{HA}$ and $\mathrm{ZnPc}$ were integrated in the same way to afford 621-AuNR-Si-HA and 621-AuNR-Si-ZnPc-HA with final absorbance around $621 \mathrm{~nm}$ (Fig. S11 and S12a, ESI $\dagger$ ). The ABDA quenching was again carried out to compare the ${ }^{1} \mathrm{O}_{2}$ production between 621-AuNR-Si-ZnPc-HA and abovementioned 711-AuNR-Si-ZnPcHA. After $10 \mathrm{~min}$ irradiation of these samples with similar gold-to-ZnPc concentrations, the relative absorbance of ABDA measured at $380 \mathrm{~nm}$ decreased by $28 \%$ for $711-\mathrm{AuNR}-\mathrm{Si}-\mathrm{ZnPc}$ HA and $11 \%$ for 621-AuNR-Si-ZnPc-HA. For Si-ZnPc-HA and free $\mathrm{ZnPc}$, the absorbance only decreased by $9 \%$ and $5 \%$, respectively (Fig. 4a). This comparison indicates that the ${ }^{1} \mathrm{O}_{2}$ production was indeed enhanced when the absorbance maxima of AuNR and the photosensitizer overlapped at around $711 \mathrm{~nm}$. The photothermal ability of 621-AuNR-Si-ZnPc-HA was tested against 711-AuNR-Si-ZnPc-HA (Fig. S12b, ESI $\dagger$ ). Lower concentration of gold than that of the previous study was utilized, and the optical density at the respective maximal wavelength was ensured to be the same. In this case, 711-AuNR-Si-ZnPc-HA showed a higher temperature rise of $16{ }^{\circ} \mathrm{C}$ as compared to that $\left(7^{\circ} \mathrm{C}\right)$ of 621-AuNR-Si-ZnPc-HA upon $730 \mathrm{~nm}$ laser irradiation. It was observed that, between the $711 \mathrm{~nm}$ nanorod pair (711AuNR-Si-ZnPc-HA and 711-AuNR-Si-HA) and the $621 \mathrm{~nm}$ nanorod pair (621-AuNR-Si-ZnPc-HA and 621-AuNR-Si-HA), the difference in the temperature rise in the first 2 min was not very obvious. After the first min of irradiation, the differences in the temperature rise between the $711 \mathrm{~nm}$ and $621 \mathrm{~nm}$ nanorod pairs were $0.1{ }^{\circ} \mathrm{C}$ and $0.8{ }^{\circ} \mathrm{C}$, respectively. After the second min, the values were $0.3{ }^{\circ} \mathrm{C}$ and $0.8{ }^{\circ} \mathrm{C}$, respectively (Tables S1-S4, ESI $\dagger$ ).

HeLa cells were seeded in a 96-well plate and fed with 621-AuNR-Si-ZnPc-HA, 711-AuNR-Si-ZnPc-HA, 621-AuNR-Si-HA, 711-AuNR-Si-HA and Si-ZnPc-HA at the same concentration. 711-AuNR-Si-ZnPc-HA constantly showed a stronger killing effect than 621-AuNR-Si-ZnPc-HA due to a better photothermal effect as well as an enhanced PDT effect of the former. To investigate the PDT enhancement in vitro, the cells were irradiated for $0,1,2$ and $3 \mathrm{~min}$ and the cell viabilities were analyzed. In the first $2 \mathrm{~min}$, the temperature rise difference between 711-AuNR-Si-ZnPc-HA and 711-AuNR-Si-HA and that between 621-AuNR-Si-ZnPc-HA and 621-AuNR-Si-HA were negligible. However, the cell viability at the first and second min for the $711 \mathrm{~nm}$ nanorods decreased by $3.7 \%$ and $3.2 \%$ respectively, whereas that of the $621 \mathrm{~nm}$ nanorods was only $0.6 \%$ and $2.2 \%$. After $3 \mathrm{~min}$ of irradiation, the cell viability difference between 711-AuNR-Si-ZnPc-HA and 711-AuNR-Si-HA was $7.6 \%$, even though the temperature rise was not obvious. This observation shows that the PDT effect was dominant. For the $621 \mathrm{~nm}$ nanorod pair after $3 \mathrm{~min}$ of irradiation, the cell viability difference between 621-AuNR-Si-ZnPc-HA and 621-AuNRSi-HA increased to $6.2 \%$, possibly due to the photothermal contribution with the temperature rise. The improved cellkilling effect of 711-AuNR-Si-ZnPc-HA over 711-AuNR-Si-HA as compared to 621-AuNR-Si-ZnPc-HA over 621-AuNR-Si-HA could be attributed to the enhanced PDT effect (Tables S1 and S5, ESI $\dagger$ ).
In conclusion, we have successfully synthesized an AuNR system to present simultaneous PDT and PTT using a single light source. ZnPc was anchored on the surface of silica coated AuNR to afford closer contact with cancer cells. By utilizing LSPR provided by AuNR, the two types of therapy have been shown to behave synergistically, and the singlet oxygen production of $\mathrm{ZnPc}$ has also been enhanced. This multifunctional system has demonstrated its excellent targeting capability to CD44 receptors on cancer cells for combinational therapy.

This research was supported by the NTU-A*STAR Silicon Technologies Centre of Excellence under program grant no. 11235100003 and the NTU-Northwestern Institute for Nanomedicine.

\section{Notes and references}

1 P. Agostinis, K. Berg, K. A. Cengel, T. H. Foster, A. W. Girotti, S. O. Gollnick, S. M. Hahn, M. R. Hamblin, A. Juzeniene, D. Kessel, M. Korbelik, J. Moan, P. Mroz, D. Nowiz, J. Piette, B. C. Willson and J. Golab, Am. Cancer Soc., 2011, 61, 250-281.

2 M. C. F. Simões, J. J. S. Sousa and A. A. C. C. Pais, Cancer Lett., 2015, 357, 8-42.

3 D. K. Chatterjee, L. S. Fong and Y. Zhang, Adv. Drug Delivery Rev., 2008, 60, 1627-1637.

4 X. Ma, Q. Qu and Y. Zhao, ACS Appl. Mater. Interfaces, 2015, 7, 10671-10676.

5 H. Shi, X. Ma, Q. Zhao, B. Liu, Q. Qu, Z. An, Y. Zhao and W. Huang, Adv. Funct. Mater., 2014, 24, 4823-4830.

6 C. S. Jin, J. F. Lovell, J. Chen and G. Zheng, ACS Nano, 2013, 7, 2541-2550.

7 D. Zhang, M. Wu, Y. Zeng, L. Wu, Q. Wang, X. Han, X. Liu and J. Liu, ACS Appl. Mater. Interfaces, 2015, 7, 8176-8187.

8 B. E. Smith, P. B. Roder, X. Zhou and P. J. Pauzauskie, Nanoscale, 2015, 7, 7115-7126.

9 J. Lin, S. Wang, P. Huang, Z. Wang, S. Chen, G. Niu, W. Li, J. He, D. Cui, G. Lu, X. Chen and Z. Nie, ACS Nano, 2013, 7, 5320-5329.

10 X. Song, C. Liang, H. Gong, Q. Chen, C. Wang and Z. Liu, Small, 2015, 1-10.

11 S. Wang, A. Riedinger, H. Li, C. Fu, H. Liu, L. Li, T. Liu, L. Tan, M. J. Barthel, G. Pugliese, F. De Donato, M. S. D. Abbusco, X. Meng, L. Manna, H. Meng and T. Pellegrino, ACS Nano, 2015, 9, 1788-1800.

12 P. Vijayaraghavan, C. H. Liu, R. Vankayala, C. S. Chiang and K. C. Hwang, Adv. Mater., 2014, 6689-6695.

13 L. Gao, J. Fei, J. Zhao, H. Li, Y. Cui and J. Li, ACS Nano, 2012, 6, 8030-8040.

14 R. Vankayala, C. C. Lin, P. Kalluru, C. S. Chiang and K. C. Hwang, Biomaterials, 2014, 35, 5527-5538.

15 S. Wang, P. Huang, L. Nie, R. Xing, D. Liu, Z. Wang, J. Lin, S. Chen, G. Niu, G. Lu and X. Chen, Adv. Mater., 2013, 25, 3055-3061.

16 W. S. Kuo, Y. T. Chang, K. C. Cho, K. C. Chiu, C. H. Lien, C. S. Yeh and S. J. Chen, Biomaterials, 2012, 33, 3270-3278.

17 Y. Li, T. Wen, R. Zhao, X. Liu, T. Ji, H. Wang, X. Shi and J. Shi, ACS Nano, 2014, 8, 11529-11542.

18 N. Wang, Z. Zhao, Y. Lv, H. Fan, H. Bai, H. Meng, Y. Long, T. Fu, X. Zhang and W. Tan, Nano Res., 2014, 7, 1291-1301.

19 H. Chen, L. Shao, Q. Li and J. Wang, Chem. Soc. Rev., 2013, 42, 2679-2724.

20 Y. Liu, Y. Liu, W. Bu, C. Cheng, C. Zuo, Q. Xiao, Y. Sun, D. Ni, C. Zhang, J. Liu and J. Shi, Angew. Chem., Int. Ed., 2015, 54, 1-6.

21 Z. Chu, C. Yin, S. Zhang, G. Lin and Q. Li, Nanoscale, 2013, 5, 3406-3411.

22 M. Li, H. Yan, C. Teh, V. Korzh and Y. Zhao, Chem. Commun., 2014, 50, 9745-9748.

23 B. Nikoobakht and M. A. El-Sayed, Chem. Mater., 2003, 15, 1957-1962.

24 W. Stöber, A. Fink and E. Bohn, J. Colloid Interface Sci., 1968, 26, 62-69.

25 M. Fujiwara, M. Akiyama, M. Hata, K. Shiokawa and R. Nomura, ACS Nano, 2008, 2, 1671-1681.

26 H. S. S. Qhattal and X. Liu, Mol. Pharmaceutics, 2011, 8, 1233-1246. 